\title{
Separation Transformation and a Class of Exact Solutions to the Higher-Dimensional Klein-Gordon-Zakharov Equation
}

\author{
Jing Chen, ${ }^{1}$ Ling Liu, ${ }^{2}$ and Li Liu ${ }^{3}$ \\ ${ }^{1}$ School of Statistics and Mathematics, Central University of Finance and Economics, Beijing 100081, China \\ ${ }^{2}$ School of Science, Beijing Information Science and Technology University, Beijing 100192, China \\ ${ }^{3}$ China Petroleum Engineering and Construction Corp., Beijing 100028, China
}

Correspondence should be addressed to Jing Chen; chenjingcufe@163.com

Received 8 February 2014; Accepted 30 March 2014; Published 24 April 2014

Academic Editor: Christian Maes

Copyright (C) 2014 Jing Chen et al. This is an open access article distributed under the Creative Commons Attribution License, which permits unrestricted use, distribution, and reproduction in any medium, provided the original work is properly cited.

\begin{abstract}
The separation transformation method is extended to the $(n+1)$-dimensional Klein-Gordon-Zakharov equation describing the interaction of the Langmuir wave and the ion acoustic wave in plasma. We first reduce the $(n+1)$-dimensional Klein-GordonZakharov equation to a set of partial differential equations and two nonlinear ordinary differential equations of the separation variables. Then the general solutions of the set of partial differential equations are given and the two nonlinear ordinary differential equations are solved by extended $F$-expansion method. Finally, some new exact solutions of the $(n+1)$-dimensional Klein-GordonZakharov equation are proposed explicitly by combining the separation transformation with the exact solutions of the separation variables. It is shown that, for the case of $n \geq 2$, there is an arbitrary function in every exact solution, which may reveal more nontrivial nonlinear structures in the high-dimensional Klein-Gordon-Zakharov equation.
\end{abstract}

\section{Introduction}

The Klein-Gordon equation (sometimes called KleinGordon-Fock equation) [1] is a relativistic version of the Schrödinger equation. Its nonlinear counterpart is the nonlinear Klein-Gordon equation [2]:

$$
\frac{\partial^{2} u}{\partial t^{2}}-\frac{\partial^{2} u}{\partial x^{2}}+\alpha u-\beta|u|^{2} u=0
$$

where $\alpha$ and $\beta$ are constants, which has important applications in various fields. For example, it is attributed to the classical $u^{4}$ field theory in the physics of elementary particles and fields, and it can describe the propagation of dislocations within crystals and the propagation of magnetic flux on a Josephson line, and so on. One extension of the nonlinear Klein-Gordon equation is the $(1+1)$-dimensional KleinGordon-Zakharov (KGZ) equation [3, 4]:

$$
\frac{\partial^{2} u}{\partial t^{2}}-\frac{\partial^{2} u}{\partial x^{2}}+\alpha u+u v-\beta|u|^{2} u=0
$$

$$
\frac{\partial^{2} v}{\partial t^{2}}-\frac{\partial^{2} v}{\partial x^{2}}=\gamma \frac{\partial^{2}|u|^{2}}{\partial x^{2}}
$$

with $u(t, x)$ as a complex function and $v(t, x)$ as a real function, which is a classical model describing the interaction of the Langmuir wave and the ion acoustic wave in plasma [3, 4 ]. The variable $u(t, x)$ denotes the fast time scale component of electric field raised by electrons and the variable $v(t, x)$ denotes the deviation of ion density from its equilibrium. In recent years, some authors applied analytical and numerical methods [5-8] to solve the (1+1)-dimensional KGZ equation (2) and obtained many exact and numerical solutions.

The high-dimensional extension of KGZ equation is important in real applications, so in this paper we would like to investigate the $(n+1)$-dimensional KGZ equation:

$$
\begin{gathered}
\frac{\partial^{2} u}{\partial t^{2}}-\Delta u+\alpha u+u v-\beta|u|^{2} u=0, \\
\frac{\partial^{2} v}{\partial t^{2}}-\Delta v+\gamma \frac{\partial^{2}|u|^{2}}{\partial t^{2}}=\gamma \Delta|u|^{2},
\end{gathered}
$$


where $\Delta=\partial^{2} / \partial x_{1}^{2}+\partial^{2} / \partial x_{2}^{2}+\cdots+\partial^{2} / \partial x_{n}^{2}=\sum_{j=1}^{n}\left(\partial^{2} / \partial x_{j}^{2}\right)$ is the Laplacian operator and $\mathbf{x} \in \mathbf{R}^{n}$. This equation is the generalization of the KGZ equation (2) and we will show that it has many exact solutions with an arbitrary function.

More recently, Wang [9] extended the separation transformation method proposed in [10-12] to the $(N+1)$ dimensional coupled nonlinear Klein-Gordon equations. Then Liu et al. [13] and we [14] further extended the separation transformation method to various high-dimensional nonlinear soliton equations and obtained explicitly many exact solutions with arbitrary functions.

In this paper, by means of the separation transformation approach [9-14] we derive the exact solutions of the $(n+$ 1)-dimensional KGZ equation (3). The rest of this paper is organized as follows. In Section 2, a separation transformation is presented and the $(n+1)$-dimensional KGZ equation (3) is reduced to a set of partial differential equations and two nonlinear ordinary differential equations. In Section 3, the two nonlinear ordinary differential equations are solved and some special exact solutions of the $(n+1)$-dimensional KGZ equation (3) are constructed explicitly. Conclusions are presented in Section 4.

\section{Separation Transformation and Its Application}

The following proposition reveals the relationship between the exact solutions of the $(n+1)$-dimensional KGZ equation (3) and two nonlinear ordinary differential equations (ODEs) along with a set of partial differential equations (PDEs).

Proposition 1. The functions $u\left(t ; x_{1}, \ldots, x_{n}\right)=U\left[\omega\left(t ; x_{1}, \ldots\right.\right.$, $\left.\left.x_{n}\right)\right] \exp \left[i X\left(t ; x_{1}, \ldots, x_{n}\right)\right]$ and $v\left(t ; x_{1}, \ldots, x_{n}\right)=V[\omega(t ;$ $\left.\left.x_{1}, \ldots, x_{n}\right)\right]$ solve the $(n+1)$-dimensional KGZ equation (3) if the functions $\omega=\omega\left(t ; x_{1}, \ldots, x_{n}\right), X=X\left(t ; x_{1}, \ldots, x_{n}\right)$, $U(\omega)=U\left[\omega\left(t ; x_{1}, \ldots, x_{n}\right)\right]$, and $V(\omega)=V\left[\omega\left(t ; x_{1}, \ldots, x_{n}\right)\right]$ solve the following set of differential equations:

$$
\begin{gathered}
\omega_{t t}-\Delta \omega=0, \\
\omega_{t}^{2}-\sum_{j=1}^{n} \omega_{x_{j}}^{2}=K_{1}, \\
X_{t t}-\Delta X=0, \\
X_{t}^{2}-\sum_{j=1}^{n} X_{x_{j}}^{2}=K_{2}, \\
\omega_{t} X_{t}-\sum_{j=1}^{n} \omega_{x_{j}} X_{x_{j}}=0, \\
V^{\prime \prime}(\omega)+\gamma\left(U^{2}(\omega)\right)^{\prime \prime}=0, \\
K_{1} U^{\prime \prime}(\omega)+\left(\alpha-K_{2}\right) U(\omega)+U(\omega) V(\omega)-\beta U^{3}(\omega)=0,
\end{gathered}
$$

where $U^{\prime \prime}=d^{2} U / d \omega^{2}, V^{\prime \prime}=d^{2} V / d \omega^{2}$, and $K_{1}>0$ and $K_{2}$ are constants.
Proof. Assume that the $(n+1)$-dimensional KGZ equation (3) has the following solution:

$$
\begin{gathered}
u\left(t ; x_{1}, \ldots, x_{n}\right) \\
=U\left[\omega\left(t ; x_{1}, \ldots, x_{n}\right)\right] \exp \left[i X\left(t ; x_{1}, \ldots, x_{n}\right)\right], \\
v\left(t ; x_{1}, \ldots, x_{n}\right)=V\left[\omega\left(t ; x_{1}, \ldots, x_{n}\right)\right],
\end{gathered}
$$

where $\omega\left(t ; x_{1}, \ldots, x_{n}\right)$ and $X\left(t ; x_{1}, \ldots, x_{n}\right)$ are functions to be determined.

Substituting (6) into the $(n+1)$-dimensional KGZ equation (3) yields two ODEs:

$$
\begin{aligned}
& \left(\omega_{t}^{2}-\sum_{j=1}^{n} \omega_{x_{j}}^{2}\right) U^{\prime \prime}(\omega)+\left(\omega_{t t}-\Delta \omega\right) U^{\prime}(\omega) \\
& -\left(X_{t}^{2}-\sum_{j=1}^{n} X_{x_{j}}^{2}\right) U(\omega)+\alpha U(\omega)+U(\omega) V(\omega) \\
& -\beta U^{3}(\omega)+2 i\left(\omega_{t} X_{t}-\sum_{j=1}^{n} \omega_{x_{j}} X_{x_{j}}\right) U^{\prime}(\omega) \\
& +i\left(X_{t t}-\Delta X\right) U(\omega)=0 \\
& \left(\omega_{t}^{2}-\sum_{j=1}^{n} \omega_{x_{j}}^{2}\right)\left[V^{\prime \prime}(\omega)+\gamma\left(U^{2}(\omega)\right)^{\prime \prime}\right] \\
& +\left(\omega_{t t}-\Delta \omega\right)\left[V^{\prime}(\omega)+\gamma\left(U^{2}(\omega)\right)^{\prime}\right]=0 .
\end{aligned}
$$

If asking $\omega\left(t ; x_{1}, \ldots, x_{n}\right)$ and $X\left(t ; x_{1}, \ldots, x_{n}\right)$ to solve

$$
\begin{gathered}
\omega_{t t}-\Delta \omega=0, \\
\omega_{t}^{2}-\sum_{j=1}^{n} \omega_{x_{j}}^{2}=K_{1}, \\
X_{t t}-\Delta X=0, \\
X_{t}^{2}-\sum_{j=1}^{n} X_{x_{j}}^{2}=K_{2}, \\
\omega_{t} X_{t}-\sum_{j=1}^{n} \omega_{x_{j}} X_{x_{j}}=0,
\end{gathered}
$$

where $K_{1}$ and $K_{2}$ are auxiliary constants, then (7) are reduced to two nonlinear ODEs of functions $U(\omega)$ and $V(\omega)$ as

$$
\begin{gathered}
V^{\prime \prime}(\omega)+\gamma\left(U^{2}(\omega)\right)^{\prime \prime}=0, \\
K_{1} U^{\prime \prime}(\omega)+\left(\alpha-K_{2}\right) U(\omega)+U(\omega) V(\omega)-\beta U^{3}(\omega)=0 .
\end{gathered}
$$

This finishes the proof of the proposition.

We see that under the separation transformation (6) the $(n+1)$-dimensional KGZ equation (3) is separated into 
two sets of differential equations, namely, the PDEs in (8) and ODEs in (9). If we can obtain the exact solutions of the differential equations (8) and (9), the explicitly exact solutions of the $(n+1)$-dimensional KGZ equation (3) can be built immediately. In what follows, we solve the PDEs in (8) firstly.

When $n=1$, the PDEs of functions $\omega\left(t ; x_{1}\right), X\left(t ; x_{1}\right)$ in (8) become

$$
\begin{gathered}
\omega_{t t}-\omega_{x_{1} x_{1}}=0, \\
\omega_{t}^{2}-\omega_{x_{1}}^{2}=K_{1}, \\
X_{t t}-X_{x_{1} x_{1}}=0, \\
X_{t}^{2}-X_{x_{1}}^{2}=K_{2}, \\
\omega_{t} X_{t}-\omega_{x_{1}} X_{x_{1}}=0,
\end{gathered}
$$

which has the following general solutions:

$$
\begin{gathered}
\omega\left(t ; x_{1}\right)=c_{1}\left(x_{1}+t\right)-\frac{K_{1}}{4 c_{1}}\left(x_{1}-t\right)+c_{2}, \\
X\left(t ; x_{1}\right)=d_{0} t+d_{1} x_{1}+d_{2},
\end{gathered}
$$

where $d_{0}^{2}=d_{1}^{2}+K_{2}, d_{0}\left(4 c_{1}^{2}+K_{1}\right)+d_{1}\left(K_{1}-4 c_{1}^{2}\right)=0$, and $c_{2}$, $d_{2}$ are integral constants.

When $n \geq 2$, the PDEs in (8) have the following general solutions:

$$
\begin{aligned}
& \omega\left(t ; x_{1}, \ldots, x_{n}\right)=f\left(\sum_{j=1}^{n} k_{j} x_{j}+k_{0} t+c_{3}\right) \\
& +\sqrt{\frac{K_{1}}{l_{0}^{2}-\sum_{j=1}^{n} l_{j}^{2}}}\left(\sum_{j=1}^{n} l_{j} x_{j}+l_{0} t+c_{4}\right), \\
& X\left(t ; x_{1}, \ldots, x_{n}\right)=\sum_{j=1}^{n} \lambda_{j} x_{j}+\lambda_{0} t+d_{3} \text {, }
\end{aligned}
$$

where $f=f(\cdot)$ is an arbitrary function, $c_{3}, c_{4}$, and $d_{3}$ are constants, and $k_{0}, k_{j}, \lambda_{0}, \lambda_{j}, l_{0}$, and $l_{j}(j=1, \ldots, n)$ are constants satisfying

$$
\begin{gathered}
k_{0}^{2}=\sum_{j=1}^{n} k_{j}^{2}, \quad k_{0} l_{0}=\sum_{j=1}^{n} k_{j} l_{j}, \quad \lambda_{0} l_{0}=\sum_{j=1}^{n} \lambda_{j} l_{j}, \\
\lambda_{0} k_{0}=\sum_{j=1}^{n} \lambda_{j} k_{j}, \quad \lambda_{0}^{2}=\sum_{j=1}^{n} \lambda_{j}^{2}+K_{2} .
\end{gathered}
$$

Thus we conclude in this section that the exact solution of the $(n+1)$-dimensional KGZ equation (3) can be written as

$$
\begin{gathered}
u\left(t ; x_{1}, \ldots, x_{n}\right) \\
=U\left[\omega\left(t ; x_{1}, \ldots, x_{n}\right)\right] \exp \left[i X\left(t ; x_{1}, \ldots, x_{n}\right)\right] \\
v\left(t ; x_{1}, \ldots, x_{n}\right)=V\left[\omega\left(t ; x_{1}, \ldots, x_{n}\right)\right]
\end{gathered}
$$

where $U(\omega)$ and $V(\omega)$ satisfy ODEs (9) and $\omega\left(t ; x_{1}, \ldots, x_{n}\right)$, $X\left(t ; x_{1}, \ldots, x_{n}\right)$ are functions given by (11) for $n=1$ and (12) with (13) for $n \geq 2$.

It is remarked that when $n \geq 2$, there is an arbitrary function $f\left(\sum_{j=1}^{n} k_{j} x_{j}+k_{0} t+c_{3}\right)$ in each exact solution of the $(n+1)$-dimensional KGZ equation (3), which may reveal abundant nonlinear structures in this nonlinear equation.

\section{New Exact Solutions of the $(n+1)$ - Dimensional KGZ Equation (3)}

In this section, we search for the exact solutions of the nonlinear ODEs in (9) by means of the F-expansion method proposed by Wang et al. [15-17]. Based on the explicit solutions of the ODEs in (11), many exact solutions of the $(n+1)$-dimensional KGZ equation (3) are obtained explicitly via the separation transformation (6).

Integrating the first equation in (9) we have

$$
V(\omega)=-\gamma U^{2}(\omega)
$$

Thus the second equation in (9) becomes an $\mathrm{ODE}$ of $U(\omega)$ as

$$
K_{1} U^{\prime \prime}(\omega)+\left(\alpha-K_{2}\right) U(\omega)-\gamma U^{3}(\omega)-\beta U^{3}(\omega)=0 .
$$

In what follows, we solve the ODE (16) by using the extended $F$-expansion method proposed by Wang et al. [1517]. In doing so, assume that the solution of ODE (16) is

$$
U(\omega)=a_{0}+a_{1} F(\omega)+\frac{b_{1}}{F(\omega)},
$$

where $a_{0}, a_{1}$, and $b_{1}$ are constants to be determined and $F(\omega)$ satisfies the elliptic equation [18]:

$$
\left(\frac{d F(\omega)}{d \omega}\right)^{2}=r+q F^{2}(\omega)+p F^{4}(\omega),
$$

whose solutions in Jacobi elliptic function forms [18] are listed in Table 1 in the Appendix.

Substituting (17) with (18) into ODE (16), we find that the variables $a_{0}, a_{1}, b_{1}$, and $\alpha$ have two groups of solutions.

Group 1. Consider that

$$
a_{0}=0, \quad b_{1}=0, \quad \alpha=K_{2}-K_{1} q, \quad a_{1}=\sqrt{\frac{2 K_{1} p}{\gamma+\beta}} .
$$

Group 2. Consider that

$$
\begin{gathered}
a_{0}=0, \quad a_{1}=\sqrt{\frac{2 K_{1} p}{\gamma+\beta}}, \quad b_{1}=\sqrt{\frac{2 K_{1} r}{\gamma+\beta}}, \\
\alpha=K_{2}-K_{1} q+6 \sqrt{\frac{K_{1} p}{\gamma+\beta}} \sqrt{\frac{K_{1} r}{\gamma+\beta}}(\gamma+\beta) .
\end{gathered}
$$

Combining the separation transformation (6) with (15), (17), (19), or (20) along with (11)-(13) and the solutions 
TABLE 1: Exact solutions of $(d f(w) / d w)^{2}=r+q F^{2}+p F^{4}(\omega)(\omega)$.

\begin{tabular}{lcccc}
\hline & $r$ & $q$ & $p$ & $F(\omega)$ \\
\hline 1 & 1 & $-\left(1+m^{2}\right)$ & $m^{2}$ & $\operatorname{sn}(\omega, m)$ \\
2 & $1-m^{2}$ & $2 m^{2}-1$ & $-m^{2}$ & $\operatorname{cn}(\omega, m)$ \\
3 & $m^{2}-1$ & $2-m^{2}$ & -1 & $\operatorname{dn}(\omega, m)$ \\
4 & $m^{2}$ & $-\left(1+m^{2}\right)$ & 1 & $n s(\omega, m)=[\operatorname{sn}(\omega, m)]^{-1}$ \\
5 & $-m^{2}$ & $2 m^{2}-1$ & $1-m^{2}$ & $n c(\omega, m)=[\mathrm{cn}(\omega, m)]^{-1}$ \\
6 & -1 & $2-m^{2}$ & $m^{2}-1$ & $\operatorname{nd}(\omega, m)=[\operatorname{dn}(\omega, m)]^{-1}$ \\
7 & 1 & $2-m^{2}$ & $1-m^{2}$ & $\operatorname{sc}(\omega, m)=\operatorname{sn}(\omega, m) / \operatorname{cn}(\omega, m)$ \\
8 & 1 & $2 m^{2}-1$ & $\operatorname{sd}(\omega, m)=\operatorname{sn}(\omega, m) / \operatorname{dn}(\omega, m)$ \\
9 & $1-m^{2}$ & $\left.1-m^{2}\right)$ & $\operatorname{cs}(\omega, m)=\operatorname{cn}(\omega, m) / \operatorname{sn}(\omega, m)$ \\
10 & 1 & $-\left(1+m^{2}\right)$ & $\operatorname{cd}(\omega, m)=\operatorname{cn}(\omega, m) / \operatorname{dn}(\omega, m)$ \\
11 & $-m^{2}\left(1-m^{2}\right)$ & $2 m^{2}-1$ & $\operatorname{ds}(\omega, m)=\operatorname{dn}(\omega, m) / \operatorname{sn}(\omega, m)$ \\
12 & $m^{2}$ & $-\left(1+m^{2}\right)$ & 1 & $\operatorname{dc}(\omega, m)=\operatorname{dn}(\omega, m) / \operatorname{cn}(\omega, m)$ \\
13 & $1 / 4$ & $\left(1-2 m^{2}\right) / 2$ & $\operatorname{ns}(\omega, m)+\operatorname{cs}(\omega, m)$ \\
14 & $\left(1-m^{2}\right) / 4$ & $\left(1+m^{2}\right) / 2$ & $\operatorname{nc}(\omega, m)+\operatorname{sc}(\omega, m)$ \\
15 & $m^{4} / 4$ & $\left(m^{2}-2\right) / 2$ & $\left(1-m^{2}\right) / 4$ & $\operatorname{ns}(\omega, m)+\operatorname{ds}(\omega, m)$ \\
\hline
\end{tabular}

of elliptic equation (18) in Table 1 yields the special exact solutions of the $(n+1)$-dimensional KGZ equation (3) as follows.

Solution 1. Jacobi elliptic sn-function solution is as follows:

$$
\begin{aligned}
& u\left(t ; x_{1}, \ldots, x_{n}\right)=m \sqrt{\frac{2 K_{1}}{\gamma+\beta}} \operatorname{sn}(\omega, m) e^{i X}, \\
& v\left(t ; x_{1}, \ldots, x_{n}\right)=-\frac{2 K_{1} m^{2} \gamma}{\gamma+\beta} \operatorname{sn}^{2}(\omega, m),
\end{aligned}
$$

where $\operatorname{sn}(\omega, m)$ is Jacobi elliptic sn-function with modulus $0 \leq m \leq 1$ and the functions $\omega$ and $X$ are given by (11) for $n=1$ and (12) with (13) for $n \geq 2$. When $m \rightarrow 1$, we have the soliton solution of the $(n+1)$-dimensional KGZ equation (3) as

$$
\begin{aligned}
& u\left(t ; x_{1}, \ldots, x_{n}\right)=\sqrt{\frac{2 K_{1}}{\gamma+\beta}} \tanh (\omega) e^{i X}, \\
& v\left(t ; x_{1}, \ldots, x_{n}\right)=-\frac{2 K_{1} \gamma}{\gamma+\beta} \tanh ^{2}(\omega) .
\end{aligned}
$$

Solution 2. Jacobi elliptic cn-function solution is as follows:

$$
\begin{aligned}
& u\left(t ; x_{1}, \ldots, x_{n}\right)=m \sqrt{-\frac{2 K_{1}}{\gamma+\beta}} \mathrm{cn}(\omega, m) e^{i X}, \\
& v\left(t ; x_{1}, \ldots, x_{n}\right)=\frac{2 K_{1} m^{2} \gamma}{\gamma+\beta} \mathrm{cn}^{2}(\omega, m),
\end{aligned}
$$

where $\mathrm{cn}(\omega, m)$ is Jacobi elliptic cn-function with modulus $0 \leq m \leq 1$ and the functions $\omega$ and $X$ are given by (11) for $n=1$ and (12) with (13) for $n \geq 2$. When $m \rightarrow 1$, we have the soliton solution of the $(n+1)$-dimensional KGZ equation (3) as

$$
\begin{aligned}
& u\left(t ; x_{1}, \ldots, x_{n}\right)=\sqrt{-\frac{2 K_{1}}{\gamma+\beta}} \operatorname{sech}(\omega) e^{i X}, \\
& v\left(t ; x_{1}, \ldots, x_{n}\right)=\frac{2 K_{1} \gamma}{\gamma+\beta} \operatorname{sech}^{2}(\omega) .
\end{aligned}
$$

Solution 3. Jacobi elliptic dn-function solution is as follows:

$$
\begin{aligned}
& u\left(t ; x_{1}, \ldots, x_{n}\right)=\sqrt{-\frac{2 K_{1}}{\gamma+\beta}} \operatorname{dn}(\omega, m) e^{i X}, \\
& v\left(t ; x_{1}, \ldots, x_{n}\right)=\frac{2 K_{1} \gamma}{\gamma+\beta} \operatorname{dn}^{2}(\omega, m),
\end{aligned}
$$

where $\operatorname{dn}(\omega, m)$ is Jacobi elliptic dn-function with modulus $0 \leq m \leq 1$ and the functions $\omega$ and $X$ are given by (11) for $n=1$ and (12) with (13) for $n \geq 2$.

Solution 4. Jacobi elliptic ns-cs-function solution is as follows:

$$
\begin{aligned}
& u\left(t ; x_{1}, \ldots, x_{n}\right)=\frac{1}{2} \sqrt{\frac{2 K_{1}}{\gamma+\beta}}[\mathrm{ns}(\omega, m)+\operatorname{cs}(\omega, m)] e^{i X}, \\
& v\left(t ; x_{1}, \ldots, x_{n}\right)=-\frac{\gamma K_{1}}{2(\gamma+\beta)}[\mathrm{ns}(\omega, m)+\operatorname{cs}(\omega, m)]^{2},
\end{aligned}
$$

where $\operatorname{ns}(\omega, m)=1 / \operatorname{sn}(\omega, m)$ and $\operatorname{cs}(\omega, m)=\operatorname{cn}(\omega$, $m) / \operatorname{sn}(\omega, m)$ are Jacobi elliptic functions with modulus $0 \leq$ $m \leq 1$ and the functions $\omega$ and $X$ are given by (11) for $n=1$ and (12) with (13) for $n \geq 2$. 
Solution 5. Jacobi elliptic nc-sc-function solution is as follows:

$$
\begin{gathered}
u\left(t ; x_{1}, \ldots, x_{n}\right) \\
=\frac{\sqrt{2}}{2} \sqrt{-\frac{K_{1}\left(m^{2}-1\right)}{\gamma+\beta}[\mathrm{nc}(\omega, m)+\mathrm{sc}(\omega, m)] e^{i X},} \\
v\left(t ; x_{1}, \ldots, x_{n}\right)=\frac{\gamma K_{1}\left(m^{2}-1\right)}{2(\gamma+\beta)}[\mathrm{nc}(\omega, m)+\operatorname{sc}(\omega, m)]^{2},
\end{gathered}
$$

wherenc $(\omega, m)=1 / \operatorname{cn}(\omega, m)$ and $\operatorname{sc}(\omega, m)=\operatorname{sn}(\omega, m) / \operatorname{cn}(\omega, m)$ are Jacobi elliptic functions with modulus $0 \leq m \leq 1$ and the functions $\omega$ and $X$ are given by (11) for $n=1$ and (12) with (13) for $n \geq 2$. Note that $m \neq 1$ here.

Solution 6. Jacobi elliptic ns-ds-function solution is as follows:

$$
\begin{aligned}
& u\left(t ; x_{1}, \ldots, x_{n}\right)=\frac{\sqrt{2}}{2} \sqrt{\frac{K_{1}}{\gamma+\beta}}[\mathrm{ns}(\omega, m)+\mathrm{ds}(\omega, m)] e^{i X}, \\
& v\left(t ; x_{1}, \ldots, x_{n}\right)=-\frac{\gamma K_{1}}{2(\gamma+\beta)}[\mathrm{ns}(\omega, m)+\mathrm{ds}(\omega, m)]^{2},
\end{aligned}
$$

where $\mathrm{ds}(\omega, m)=\operatorname{dn}(\omega, m) / \operatorname{sn}(\omega, m)$ is a Jacobi elliptic function with modulus $0 \leq m \leq 1$ and the functions $\omega$ and $X$ are given by (11) for $n=1$ and (12) with (13) for $n \geq 2$.

Solution 7. Jacobi elliptic sn-ns-function solution is as follows:

$$
\begin{aligned}
& u\left(t ; x_{1}, \ldots, x_{n}\right)=\sqrt{\frac{2 K_{1}}{\gamma+\beta}}[m \operatorname{sn}(\omega, m)+\mathrm{ns}(\omega, m)] e^{i X}, \\
& v\left(t ; x_{1}, \ldots, x_{n}\right)=-\frac{2 \gamma K_{1}}{\gamma+\beta}[m \operatorname{sn}(\omega, m)+\mathrm{ns}(\omega, m)]^{2},
\end{aligned}
$$

where the functions $\omega$ and $X$ are given by (11) for $n=1$ and (12) with (13) for $n \geq 2$.
Solution 8. Jacobi elliptic cn-nc-function solution is as follows:

$$
\begin{aligned}
& u\left(t ; x_{1}, \ldots, x_{n}\right)=\left[m \sqrt{-\frac{2 K_{1}}{\gamma+\beta}} \mathrm{cn}(\omega, m)\right.\left.\sqrt{\frac{2 K_{1}}{\gamma+\beta}\left(1-k^{2}\right)} \operatorname{nc}(\omega, m)\right] e^{i X}, \\
& v\left(t ; x_{1}, \ldots, x_{n}\right)=-\gamma\left[m \sqrt{-\frac{2 K_{1}}{\gamma+\beta}} \mathrm{cn}(\omega, m)\right. \\
&+\sqrt{\left.\frac{2 K_{1}}{\gamma+\beta}\left(1-k^{2}\right) \operatorname{nc}(\omega, m)\right]^{2},}
\end{aligned}
$$

where the functions $\omega$ and $X$ are given by (11) for $n=1$ and (12) with (13) for $n \geq 2$.

Solution 9. Jacobi elliptic dn-nd-function solution is as follows:

$$
\begin{aligned}
& u\left(t ; x_{1}, \ldots, x_{n}\right)= {\left[\sqrt{-\frac{2 K_{1}}{\gamma+\beta}} \mathrm{dn}(\omega, m)\right.} \\
&\left.+\sqrt{\frac{2 K_{1}}{\gamma+\beta}\left(m^{2}-1\right)} \operatorname{nd}(\omega, m)\right] e^{i X}, \\
& v\left(t ; x_{1}, \ldots, x_{n}\right)=-\gamma\left[\sqrt{-\frac{2 K_{1}}{\gamma+\beta}} \operatorname{dn}(\omega, m)\right. \\
&\left.+\sqrt{\frac{2 K_{1}}{\gamma+\beta}\left(m^{2}-1\right)} \operatorname{nd}(\omega, m)\right]^{2},
\end{aligned}
$$

where $\operatorname{nd}(\omega, m)=1 / \operatorname{dn}(\omega, m)$ is a Jacobi elliptic function with modulus $0 \leq m \leq 1$ and the functions $\omega$ and $X$ are given by (11) for $n=1$ and (12) with (13) for $n \geq 2$.

Remark 2. It is noted that we can also list many other types of exact solutions for the $(n+1)$-dimensional KGZ equation (3) by using the exact solutions of the elliptic equation (18) in Table 1.

When $n=2$, denote $x_{1}=x$ and $x_{2}=y$; we find that (3) becomes the $(2+1)$-dimensional KGZ equation as

$$
\begin{gathered}
\frac{\partial^{2} u}{\partial t^{2}}-\left(\frac{\partial^{2}}{\partial x^{2}}+\frac{\partial^{2}}{\partial y^{2}}\right) u+\alpha u+u v-\beta|u|^{2} u=0 \\
\frac{\partial^{2} v}{\partial t^{2}}-\left(\frac{\partial^{2}}{\partial x^{2}}+\frac{\partial^{2}}{\partial y^{2}}\right) v+\gamma \frac{\partial^{2}|u|^{2}}{\partial t^{2}}=\gamma\left(\frac{\partial^{2}}{\partial x^{2}}+\frac{\partial^{2}}{\partial y^{2}}\right)|u|^{2},
\end{gathered}
$$

which has separation solution of the form

$$
\begin{aligned}
& u(t, x, y)=U[\omega(t, x, y)] \exp [i X(t, x, y)], \\
& v(t, x, y)=V[\omega(t, x, y)]
\end{aligned}
$$




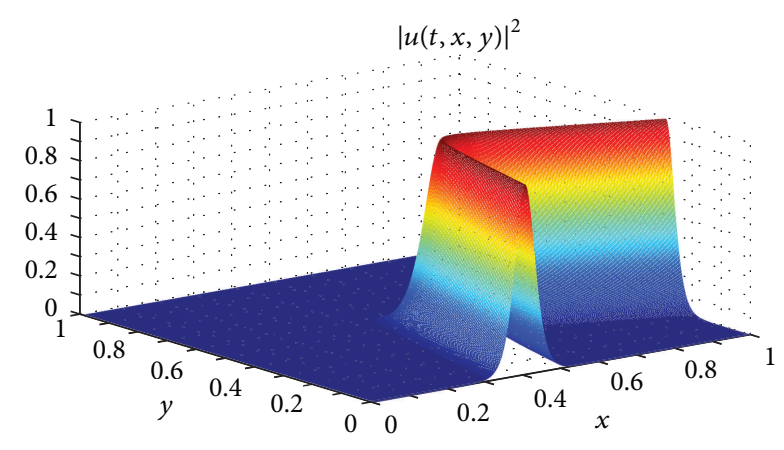

FIGURE 1: Nonlinear soliton structures of the $(2+1)$-dimensional KGZ equation at time $t=0$, given by (33)-(34) and (24) with parameters in (36) and function $f(\eta)$ satisfying (37).

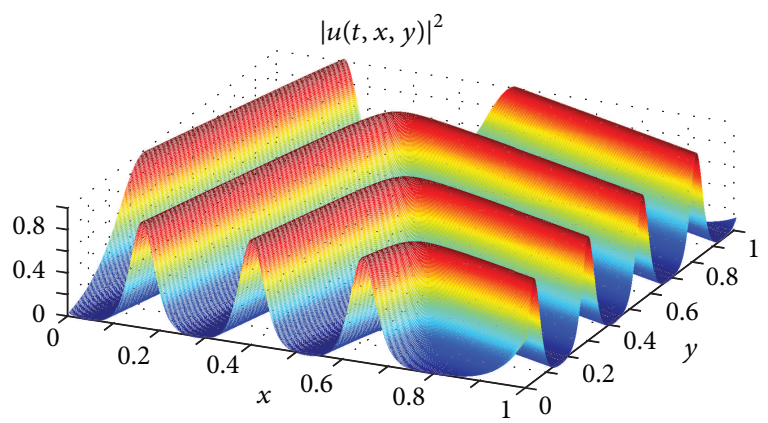

FIGURE 2: Nonlinear periodic-wave structure of the $(2+1)$ dimensional KGZ equation at time $t=0$, given by (33)-(34) and (23) with parameters in (36) and function $f(\eta)$ satisfying (37) and elliptic modulus $m=0.98$.

where $U[\omega(t, x, y)]$ and $V[\omega(t, x, y)]$ are expressed by Solutions 1-9 above and $\omega(t, x, y)$ and $X(t, x, y)$ satisfy

$$
\begin{aligned}
\omega(t ; x, y)= & f\left(k_{1} x+k_{2} y+k_{0} t+c_{3}\right) \\
& +\sqrt{\frac{K_{1}}{l_{0}^{2}-l_{1}^{2}-l_{2}^{2}}}\left(l_{1} x+l_{2} y+l_{0} t+c_{4}\right), \\
X(t ; x, y)= & \lambda_{1} x+\lambda_{2} y+\lambda_{0} t+d_{3},
\end{aligned}
$$

where $c_{3}, c_{4}$, and $d_{3}$ are constants and $k_{0}, k_{1}, k_{2}, \lambda_{0}, \lambda_{1}, \lambda_{2}, l_{0}$, $l_{1}$, and $l_{2}$ are constants satisfying

$$
\begin{gathered}
k_{0}^{2}=k_{1}^{2}+k_{2}^{2}, \quad k_{0} l_{0}=k_{1} l_{1}+k_{2} l_{2}, \quad \lambda_{0} l_{0}=\lambda_{1} l_{1}+\lambda_{2} l_{2}, \\
\lambda_{0} k_{0}=\lambda_{1} k_{1}+\lambda_{2} k_{2}, \quad \lambda_{0}^{2}=\lambda_{1}^{2}+\lambda_{2}^{2}+K_{2} .
\end{gathered}
$$

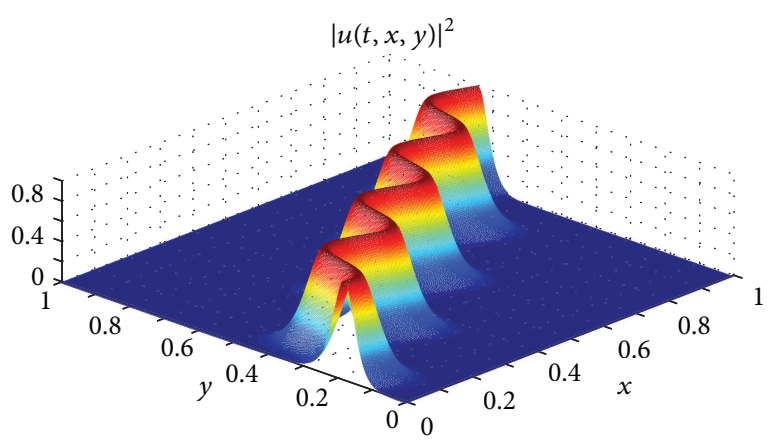

FIgURE 3: Nonlinear soliton-like structure of the $(2+1)$-dimensional KGZ equation at time $t=0$, given by (33)-(34) and (23) with function $f(\eta)$ satisfying (39).

In what follows, we take Solution 2 in (23)-(24) as an example to study the novel nonlinear structures in $(2+1)$ dimensional KGZ equation. To do so, choose a set of special solution of (35) as

$$
\begin{gathered}
K_{2}=0, \quad k_{0}=2, \quad k_{1}=\frac{\sqrt{15}}{2}, \quad k_{2}=\frac{1}{2}, \\
l_{0}=\frac{9}{4}-\frac{5 \sqrt{15}}{2}, \quad l_{1}=-10, \quad l_{2}=9, \\
\lambda_{0}=4, \quad \lambda_{1}=\sqrt{15}, \quad \lambda_{2}=1 .
\end{gathered}
$$

Because $f\left(k_{1} x+k_{2} y+k_{0} t+c_{3}\right)=f(\eta)$ is an arbitrary function of $\eta$, we can select special form of $f(\eta)$ to describe the nonlinear excitations of the $(2+1)$-dimensional KGZ equation.

Figures 1 and 2 show particular nonlinear wave structures at time $t=0$, given by (33)-(34), (23), and (24) with (36) and the function selection

$$
f(\eta)=\sqrt{\eta^{2}+1}
$$

and the choice of other parameters

$$
\begin{aligned}
& K_{1}=-1, \quad \gamma=1, \quad \beta=1, \\
& c_{3}=0, \quad c_{4}=0, \quad d_{3}=1 .
\end{aligned}
$$

Figure 3 demonstrates a particular nonlinear wave structure at time $t=0$, given by (33)-(34) and (24) with parameters in (36) and (38) and the function selection

$$
f(\eta)=\sin (\eta+1) .
$$

It is observed from Figure 3 that the special choice of arbitrary function $f(\eta)$ reveals a nonlinear soliton-like structure in the $(2+1)$-dimensional KGZ equation, which is periodic in the direction of plane $k_{1} x+k_{2} y$.

\section{Conclusion}

In conclusion, we have derived some exact Jacobi elliptic function solutions and soliton solutions of the $(n+1)$ dimensional Klein-Gordon-Zakharov equation by separation 
transformation method proposed in $[9,10,12]$. It is shown that, for the high-dimensional case, that is, $n \geq 2$, there is an arbitrary function in every exact solution of the $(n+1)$ dimensional Klein-Gordon-Zakharov equation. For the case of $n=2$ we demonstrate some novel nonlinear structures by choosing the arbitrary function $f\left(k_{1} x+k_{2} y+k_{0} t+c_{3}\right)$ specially. The separation transformation method may also be useful to solve other nonlinear wave models to explain the nonlinear excitations and localized nonlinear wave structures [19-23] in the physics of elementary particles and fields.

\section{Appendix}

In the Appendix, we present the relationships between the values of $(p, q, r)$ and the corresponding solutions of the elliptic equation (18) in Table 1; see also [18].

In the following table, the functions $\operatorname{sn}(\omega, m), \operatorname{cn}(\omega, m)$, and $\operatorname{dn}(\omega, m)$ are three basic Jacobi elliptic functions with modulus $0 \leq m \leq 1$. As seen in Table 1 , the other Jacobi elliptic functions are the combinations of these three Jacobian elliptic functions. For example, as shown before we have $\operatorname{cs}(\omega, m)=\operatorname{cn}(\omega, m) / \operatorname{sn}(\omega, m)$. When the modulus $m=1$, the Jacobi elliptic functions degenerate as the hyperbolic functions: $\operatorname{sn}(\omega, 1)=\tanh (\omega), \operatorname{cn}(\omega, 1)=\operatorname{sech}(\omega)$, and $\operatorname{dn}(\omega, 1)=\operatorname{sech}(\omega)$. When the modulus $m=0$, they degenerate as the trigonometric functions or constant: $\operatorname{sn}(\omega, 0)=\sin (\omega), \operatorname{cn}(\omega, 0)=\cos (\omega)$, and $\operatorname{dn}(\omega, 0)=1$.

\section{Conflict of Interests}

The authors declare that there is no conflict of interests regarding the publication of this paper.

\section{Acknowledgments}

This work is supported by Talent Youth Program of Beijing Municipal Commission of Education (Grant no. YETP0984), Scientific Research Common Program of Beijing Municipal Commission of Education (Grant no. SQKM201211232017), and Excellent Talent Program of Beijing (Grant no. 2012D005007000005).

\section{References}

[1] C. Itzykson and J. B. Zuber, Quantum Field Theory, McGrawHill, New York, NY, USA, 1980.

[2] V. V. Tsegel'nik, "Self-similar solutions of a system of two nonlinear partial differential equations," Differential Equations, vol. 36, no. 3, pp. 480-482, 2000.

[3] S. G. Thornhill and D. ter Haar, "Langmuir turbulence and modulational instability," Physics Reports, vol. 43, no. 2, pp. 4399, 1978.

[4] R. O. Dendy, Plama Dynamics, Oxford University Press, Oxford, UK, 1990.

[5] Y.-P. Wang and D.-F. Xia, "Generalized solitary wave solutions for the Klein-Gordon-Schrödinger equations," Computers \& Mathematics with Applications, vol. 58, no. 11-12, pp. 2300-2306, 2009.
[6] Y. Shang, Y. Huang, and W. Yuan, "New exact traveling wave solutions for the Klein-Gordon-Zakharov equations," Computers \& Mathematics with Applications, vol. 56, no. 5, pp. 14411450,2008

[7] S. Liu, Z. Fu, S. Liu, and Z. Wang, "The periodic solutions for a class of coupled nonlinear Klein-Gordon equations," Physics Letters: A, vol. 323, no. 5-6, pp. 415-420, 2004.

[8] T. Wang, J. Chen, and L. Zhang, "Conservative difference methods for the Klein-Gordon-Zakharov equations," Journal of Computational and Applied Mathematics, vol. 205, no. 1, pp. 430-452, 2007.

[9] D.-S. Wang, "A class of special exact solutions of some high dimensional non-linear wave equations," International Journal of Modern Physics B: Condensed Matter Physics. Statistical Physics. Applied Physics, vol. 24, no. 23, pp. 4563-4579, 2010.

[10] D.-S. Wang, Z. Yan, and H. Li, "Some special types of solutions of a class of the $(N+1)$-dimensional nonlinear wave equations," Computers \& Mathematics with Applications, vol. 56, no. 6, pp. 1569-1579, 2008.

[11] Z. Yan, "Separation transformation and envelope solutions of the higher-dimensional complex nonlinear Klein-Gordon equation," Physica Scripta, vol. 75, no. 3, pp. 320-322, 2007.

[12] Z. Yan, "Similarity transformations and exact solutions for a family of higher-dimensional generalized Boussinesq equations," Physics Letters: A, vol. 361, no. 3, pp. 223-230, 2007.

[13] Y. Liu, J. Chen, W. Hu, and L.-L. Zhu, "Separation transformation and new exact solutions for the $(1+\mathrm{N})$-dimensional triple Sine-Gordon equation," Zeitschrift fur Naturforschung A: Journal of Physical Sciences, vol. 66, no. 1-2, pp. 19-23, 2011.

[14] Y. Tian, J. Chen, and Z.-F. Zhang, "Separation transformation and new exact solutions of the $(N+1)$-dimensional dispersive double sine-Gordon equation," Communications in Theoretical Physics, vol. 58, no. 3, pp. 398-404, 2012.

[15] D. Wang and H.-Q. Zhang, "Further improved F-expansion method and new exact solutions of Konopelchenko-Dubrovsky equation," Chaos, Solitons and Fractals, vol. 25, no. 3, pp. 601610, 2005.

[16] D. Wang and H.-Q. Zhang, "Symbolic computation and nontravelling wave solutions of the $(2+1)$-dimensional korteweg de vries equation," Zeitschrift fur Naturforschung A: Journal of Physical Sciences, vol. 60, no. 4, pp. 221-228, 2005.

[17] M. Wang and X. Li, "Applications of F-expansion to periodic wave solutions for a new Hamiltonian amplitude equation," Chaos, Solitons and Fractals, vol. 24, no. 5, pp. 1257-1268, 2005.

[18] M. Abramowitz and I. A. Stegun, Hand Book of Mathematical Functions, Dover, New York, NY, USA, 1965.

[19] D.-S. Wang, X.-H. Hu, J. Hu, and W. M. Liu, "Quantized quasi-two-dimensional Bose-Einstein condensates with spatially modulated nonlinearity," Physical Review A-Atomic, Molecular, and Optical Physics, vol. 81, no. 2, Article ID 025604, 2010.

[20] D.-S. Wang, S.-W. Song, B. Xiong, and W. M. Liu, "Quantized vortices in a rotating Bose-Einstein condensate with spatiotemporally modulated interaction," Physical Review A-Atomic, Molecular, and Optical Physics, vol. 84, no. 5, Article ID 053607, 2011.

[21] D. S. Wang, X. Zeng, and Y. Q. Ma, "Exact vortex solitons in a quasi-two-dimensional Bose- Einstein condensate with spatially inhomogeneous cubic-quintic nonlinearity," Physics Letters A, vol. 376, no. 45, pp. 3067-3070, 2012. 
[22] D. S. Wang and X. G. Li, "Localized nonlinear matter waves in a Bose C Einstein condensate with spatially inhomogeneous two- and three-body interactions," Journal of Physics B: Atomic, Molecular and Optical Physics, vol. 45, no. 10, Article ID 105301, 2012.

[23] D. S. Wang, Y. R. Shi, K. W. Chow, Z. X. Yu, and X. G. Li, "Matterwave solitons in a spin-1 Bose-Einstein condensate with timemodulated external potential and scattering lengths," European Physical Journal D, vol. 67, article 242, 2013. 


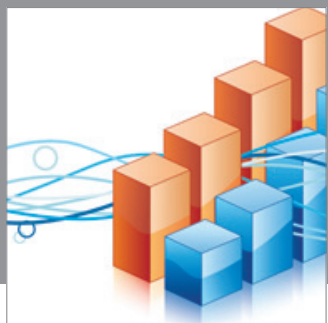

Advances in

Operations Research

mansans

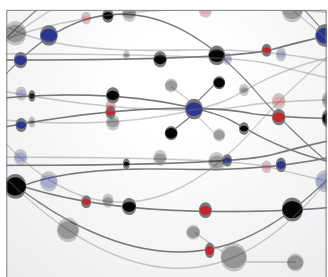

The Scientific World Journal
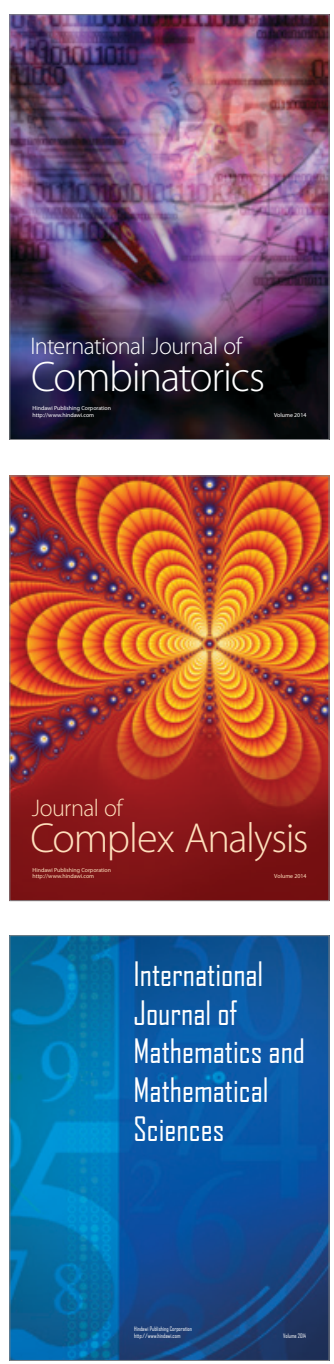
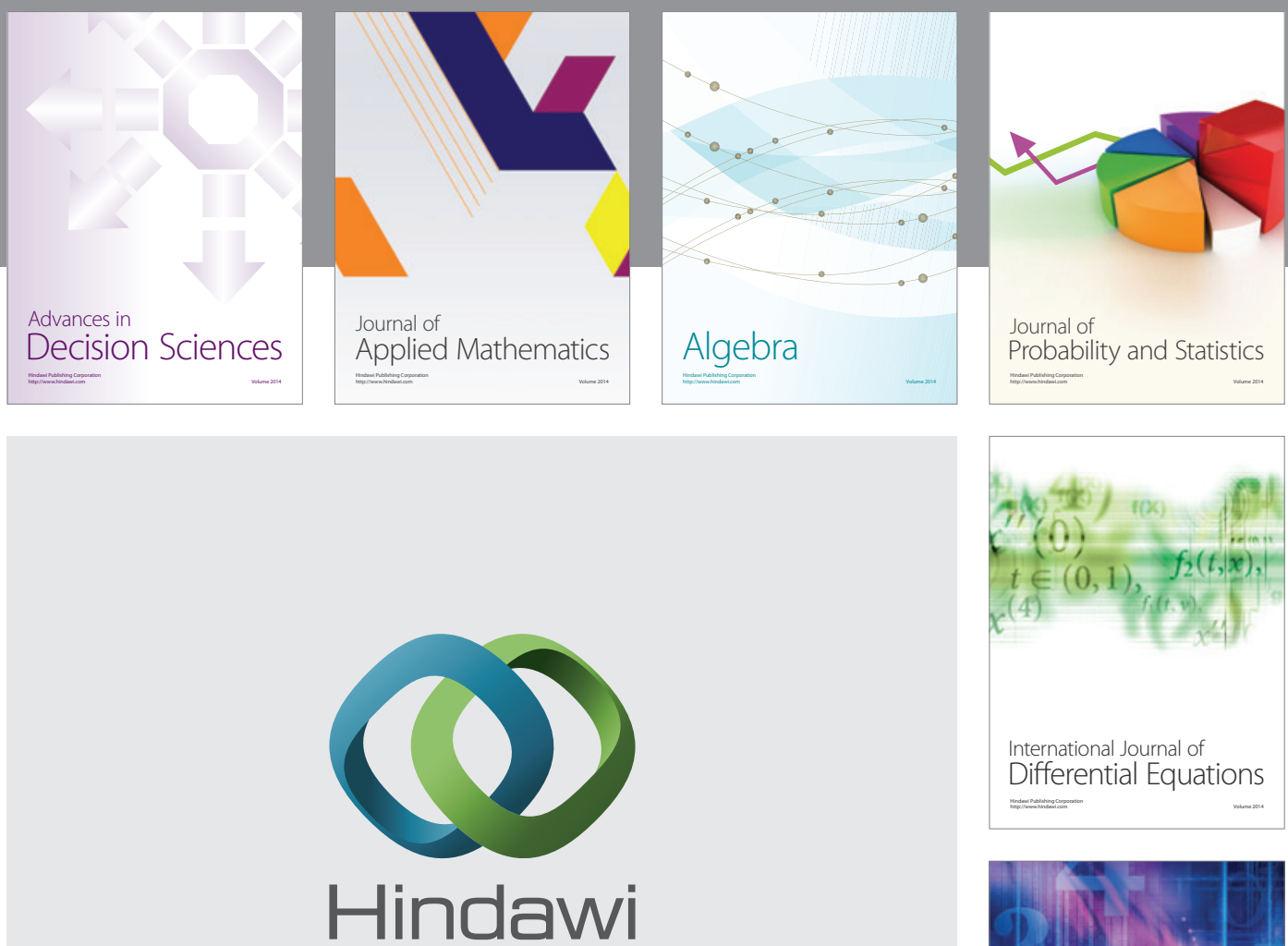

Submit your manuscripts at http://www.hindawi.com
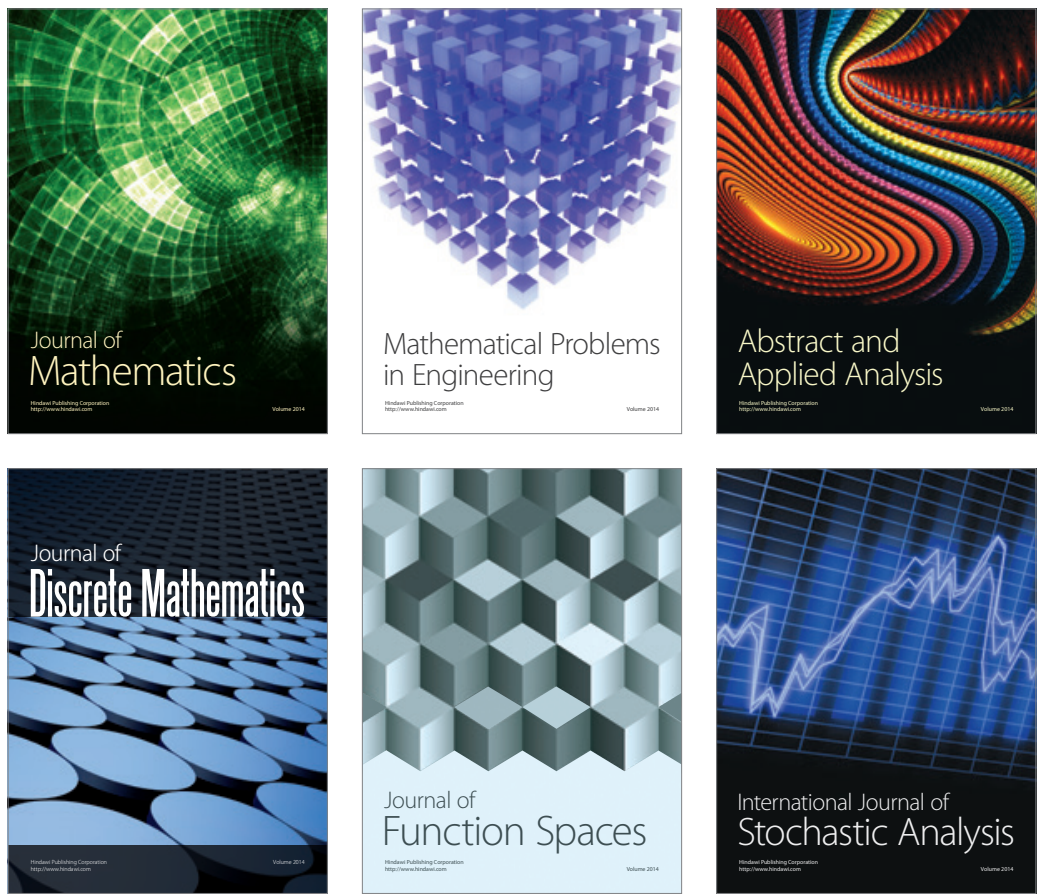

Journal of

Function Spaces

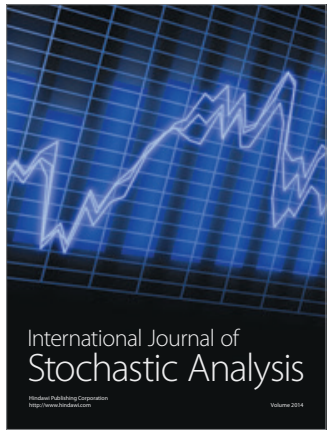

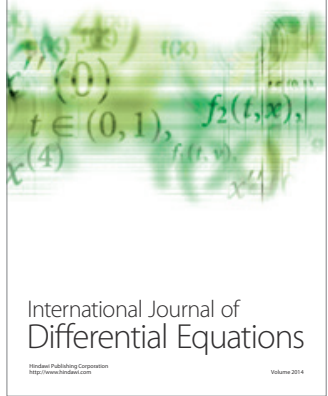
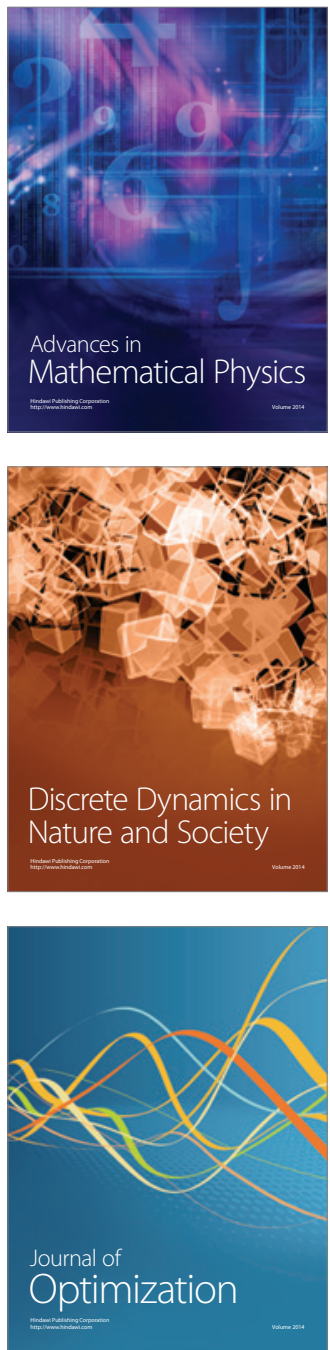